\title{
Hereditary thrombophilias and their role in the etiology of cerebral venous thrombosis
}

\author{
Ioana Cociasu ${ }^{1,2}$, Eugenia Irene Davidescu ${ }^{1,2}$, Ovidiu Alexandru Bajenaru ${ }^{1,3}$ \\ 1"Carol Davila" University of Medicine and Pharmacy, Bucharest, Romania \\ ${ }^{2}$ Neurology Clinic, Colentina Clinical Hospital, Bucharest, Romania \\ ${ }^{3}$ Neurology Clinic, Emergency University Hospital Bucharest, Romania
}

\begin{abstract}
Cerebral venous thrombosis (CVT) is a very rare and particular type of stroke. This disease mainly affects young adults and especially women of childbearing age. We performed a retrospective observational study in which we included all consecutive patients admitted with the diagnosis of cerebral venous thrombosis in the Neurology Department of Colentina Clinical Hospital between October 2013 and May 2017. Inclusion criteria were imaging confirmation of cerebral venous thrombosis either by conventional angiography, computed tomography (CT) with contrast or magnetic resonance imaging (MRI) with venography. Exclusion criteria were limited to age under 18 years old. We performed this study in order to show the genetic profile of the Romanian patient with cerebral venous thrombosis. It is the first prospective observational study performed on this disease in Romania. The results of our study concerning hereditary thrombophilia were in agreement with current data in the literature, especially given the fact that all patients presented at least one mutation. The population of patients we enrolled showed clinical and genetic aspects similar to the known epidemiologic profile of the patient with cerebral venous thrombosis, regarding age of onset, prior or concomitant infections, presence of prothrombotic factors and favourable outcome.
\end{abstract}

Keywords: cerebral venous thrombosis, epidemiology, Romania, prospective observational study, hereditary thrombophilia, genetics

\section{INTRODUCTION AND OBJECTIVES}

Cerebral venous thrombosis (CVT) is a very rare and particular type of stroke. This disease mainly affects young adults and especially women of childbearing age. According to available data, women are three times more likely to be affected by this disease than men are. On average, the incidence of cerebral venous thrombosis is significantly lower than that of arterial stroke, with values ranging between 0.22 and 1.57 cases per 100,000 inhabitants yearly. Data from the literature also suggests that incidence has been rising in the last decade, probably due to increased access to high performance imaging studies (1-5).

This disease has a very diverse range of signs and symptoms, hence the diagnosis is very often missed due to low clinical suspicion and absence of specific changes on routine imaging studies $(6,7)$.
The particular anatomy of the cerebral veins contributes to the atypical aspect of this disease. The cerebral venous system is divided in deep cerebral veins and superficial ones. The veins have thin walls, they lack valves and have no muscular tissue. Venous sinuses develop due to the folding of dural layers with the subsequent appearance of a canal $(8,9)$.

Risk factors for the appearance of cerebral venous thrombosis differ from those of arterial stroke. Historically, the most frequent causes of CVT were local infections that spread to the adjacent venous sinuses. Nowadays, due to better healthcare and improvement of antibiotic treatments these cases are sparse. Female gender and gender specific risk factors (pregnancy, puerperal period and hormone replacement therapies), malignancy and hereditary thrombophilias are the most frequent factors in- 
volved in the development of cerebral venous thrombosis. Even though the interest of the medical community for this disease has grown steadily over the years and data regarding the development of hypercoagulable states is growing, up to $35 \%$ of cases are left without an identifiable cause $(1,6,7,10)$.

Hereditary thombophilias and their role in the etiology of cerebral venous thrombosis is a hot topic at the moment. According to the results of the International Study on Cerebral Vein and Dural Sinus Thrombosis Study conducted by Ferro and collaborators, $34 \%$ of patients enrolled had a hypercoagulable state due to an inherited or acquired factor. The most high risk instances for the development of cerebral venous thrombosis are: deficiencies of natural anticoagulant proteins $(\mathrm{C}$ and $\mathrm{S})$, the deficiency of antithrombin III, the presence of antiphospholipid syndrome, high levels of homocysteine in the blood and genetic mutations that alter coagulation such as prothrombin mutation or factor V Leiden mutation. A role in the etiology of cerebral venous thrombosis is also played by mutations of methyl tetrahydrofolate reductase (MTHFR) by raising homocysteine levels in the blood. The American Heart Association (AHA) guidelines for cerebral venous thrombosis provide information regarding the severity of thrombophilia according to type, calling deficits of protein C, S or antithrombin, or homozygous mutations of prothrombin or factor V Leiden severe thrombophilias $(6,11)$.

The length of anticoagulant treatment is currently under debate. A consensus has not been reached yet whether testing for hereditary thrombophilias in all patients diagnosed with cerebral venous thrombosis would be beneficial. Nevertheless the 2010 EFNS guidelines recommend that anticoagulation length be determined taking in consideration the presence or absence of hereditary thrombophilias. Similarly, the AHA recommends that treatment duration be individualized after analysing each case for the presence of hereditary thrombophilias and other predisposing risk factors. Hence, it is surprising that after issuing these recommendations for personalized treatment regimens, the recommendation for testing patients for hereditary thrombophilia is given as being beneficial but not mandatory and the ideal time for testing is placed 2-4 weeks after finishing anticoagulation $(11,12)$.

\section{MATERIALS AND METHODS}

\section{Study design and cohort}

We performed a retrospective observational study in which we included all consecutive patients admitted with the diagnosis of cerebral venous thrombosis in the Neurology Department of Colentina Clinical Hospital between October 2013 and May 2017. Inclusion criteria were imaging confirmation of cerebral venous thrombosis either by conventional angiography, computed tomography (CT) with contrast or magnetic resonance imaging (MRI) with venography. Exclusion criteria was limited to age under 18 years old.

All patients were enrolled after the purpose of the study was explained and patients signed a written informed consent form. The study was initiated after receiving approval from the Ethics Committee of Colentina Clinical Hospital. Patients included in the study offered information regarding prior illnesses, concomitant ones and family history. They were submitted to a physical, neurological examination and cerebral imaging studies. We recorded the following parameters for all patients: age, gender, place of residence, prior illnesses, medication prior to current illness, risk factors like smoking, presence of seizures at onset or during hospitalization and location of thrombosis. Routine blood tests were performed and also specific testing for protein $\mathrm{C}$ activity, protein $\mathrm{S}$ activity, antithrombin III activity, homocysteine level. We performed genetic testing for the presence of mutations of factor II (G20210A), factor V Leiden, factor XIII, plasminogen activator inhibitor (PAI-1), MTHFR C677T and MTHFR A1298C.

\section{Data analysis}

Data obtained was recorded with Microsoft Office Excel 2013. Statistic analysis was performed using IBM SPSS Statistics for Windows version 23. Data obtained was considered to be statistically significant at a $\mathrm{p}$ value of less than 0.05 . Continuous variables with normal distribution were tested for mean value and standard deviation. Numeric variables were analysed with student's t-test and ANOVA test, whereas the Fisher test was applied for categorical variables. Sample representativeness was calculated using the Chi-squared test. We also used correlation analysis to evaluate relation- 
ships between parameters such as Pearson method for continuous variables, with normal distribution and the Spearman method for continuous variables with abnormal distribution.

\section{RESULTS}

We enrolled 23 patients with cerebral venous thrombosis, men and women equally represented. Mean age of patients was $45 \pm 16$ years old; most patients came from urban areas (88.2\%). The majority of patients reported having pre-existing illnesses $(78.9 \%)$, most of cardiovascular or neurological nature. We analysed the presence of risk factors and found smoking to be the most common (35\%). Demographic data and clinical characteristics of our study population are shown in Table 1.

TABLE 1. Demographic data and clinical characteristics of study population

\begin{tabular}{|c|c|c|}
\hline & Patients & p value \\
\hline Age (mean \pm SD) & $45 \pm 16$ & \\
\hline Female gender (n, \%) & $12(52.2 \%)$ & \\
\hline Living in urban areas (\%) & $88.2 \%$ & \\
\hline \multicolumn{3}{|l|}{ Clinical characteristics } \\
\hline Pre-exis ng illnesses (\%) & $78.9 \%$ & \\
\hline Cardiovascular disease(\%) & $46.7 \%$ & \\
\hline Neurologic disease (\%) & $40 \%$ & \\
\hline Recent infec ons (\%) & $26.7 \%$ & \\
\hline Smoking (\%) & $35 \%$ & \\
\hline Puerperal period & $18.2 \%$ & \\
\hline Deaths & $13 \%$ & \\
\hline Protein $C$ ac vity (mean $\pm S D$ ) & $96 \pm 56$ & 0.770 \\
\hline Protein $S$ ac vity (mean $\pm S D)$ & $79.6 \pm 34.8$ & 0.243 \\
\hline An thrombin III (mean $\pm S D)$ & $106.7 \pm 13.8$ & 0.608 \\
\hline Homocysteine levels (medie \pm DS) & $13.4 \pm 4.8$ & 0.508 \\
\hline
\end{tabular}

In our patient population, genetic analysis showed all patients were found to have at least one genetic mutation, as can be seen in Table 2. The most frequently found mutations were of MTHFR C677T (73.9\%), of plasminogen activator inhibitor (63.6\%) and of factor XIII, the first two reaching statistic significance. All mutations and their schematic representation in our population is portrayed in Graph 1. As to allele distribution, most our patients presented a heterozygous form, with the exception of MTHFR C677T, PAI-1 and factor XIII which were also found in homozygous form.
TABLE 2. Genetic mutations found in our patient population according to type and statistic significance

\begin{tabular}{|c|c|c|c|}
\hline Mutation & $\begin{array}{l}\text { Percentage } \\
\text { of patients }\end{array}$ & Mutation type & $\begin{array}{l}\text { Statistic } \\
\text { significance }\end{array}$ \\
\hline Prothrombin & $8.2 \%$ & $\begin{array}{c}100 \% \\
\text { heterozygote }\end{array}$ & $\begin{array}{l}p=0.949 \\
\text { Chi }(1)=0.04 \\
\text { Phi }=0.013\end{array}$ \\
\hline $\begin{array}{l}\text { Factor V } \\
\text { Leiden }\end{array}$ & $13 \%$ & $\begin{array}{c}100 \% \\
\text { heterozygote }\end{array}$ & $\begin{array}{l}p=0.484 \\
\text { Chi }(1)=0.5 \\
\text { Phi }=0.146\end{array}$ \\
\hline $\begin{array}{l}\text { MTHFR } \\
\text { C677T }\end{array}$ & $73.9 \%$ & $\begin{array}{c}82.4 \% \\
\text { heterozygote, } \\
17.6 \% \\
\text { homozygote }\end{array}$ & $\begin{array}{l}p=0.901, \\
\text { Chi }(1)=0.015, \\
\text { Phi }=0.026\end{array}$ \\
\hline $\begin{array}{l}\text { MTHFR } \\
\text { A1298C }\end{array}$ & $43.5 \%$ & $\begin{array}{c}100 \% \\
\text { heterozygote }\end{array}$ & $\begin{array}{l}p=0.034 \\
\text { Chi }(1)=0.015, \\
\text { Phi }=0.038\end{array}$ \\
\hline PAI-1 & $63.6 \%$ & $\begin{array}{c}57.1 \% \\
\text { heterozygote, } \\
42.9 \% \\
\text { homozygote }\end{array}$ & $\begin{array}{l}\mathbf{p}=\mathbf{0 . 0 1 9} \\
\text { Chi }(1)=5.5 \\
\text { Phi }=0.5\end{array}$ \\
\hline Factor XIII & $45.5 \%$ & $\begin{array}{c}70 \% \\
\text { heterozygote, } \\
\text { 30\% homozygote }\end{array}$ & $\begin{array}{l}p=0.184, \\
\text { Chi }(1)=1.766, \\
\text { Phi }=0.283\end{array}$ \\
\hline
\end{tabular}

\section{Mutation distribution within cohort}

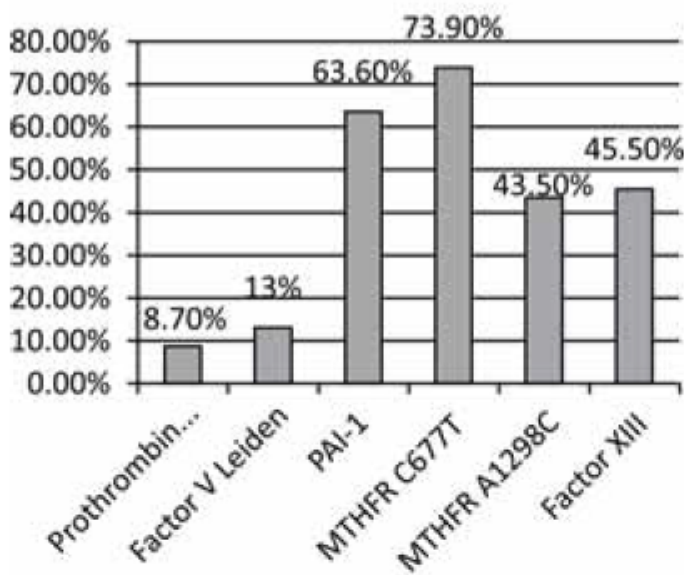

$\square$ patients with mutation present

GRAPH 1. Percentage of carriers of each mutation in our patient population

We analysed homocysteine blood levels in our patients and found the mean value exceeded the normal upper limit $(<10 \mu \mathrm{mol} / \mathrm{L})$. This increase in homocysteine mean value was due to the fact that most males included in the study had hyperhomcysteinemia $(p=0.508)$. The values of homocysteine and their distribution by gender can be seen in Graph 2. The graph also shows that $75 \%$ of males showed values above the normal upper limit. 


\section{Homocysteine levels}

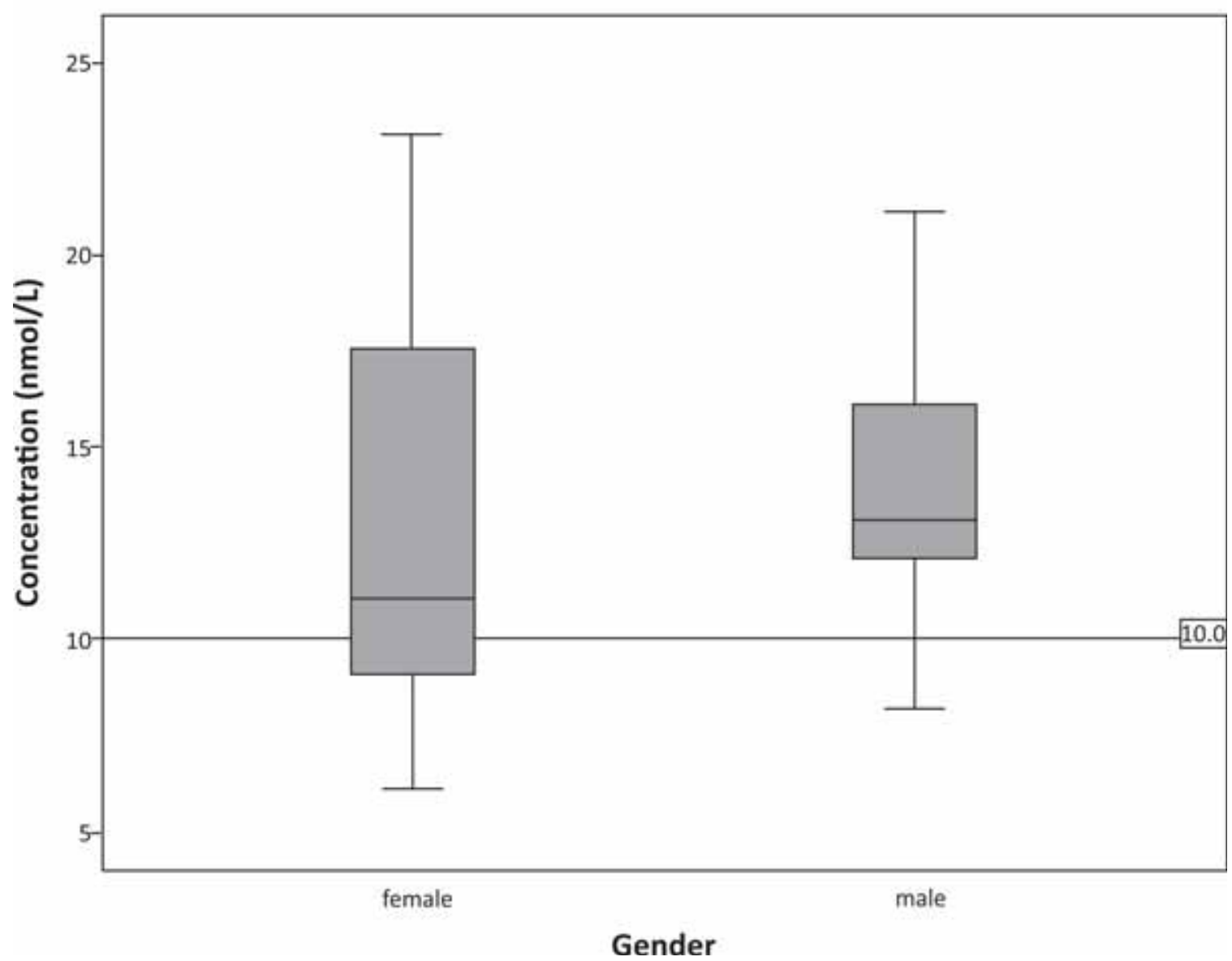

GRAPH 2. Distribution of homocysteine levels by gender

$13 \%$ of our patients were found to have factor $\mathrm{V}$ Leiden mutation. All cases presented a heterozygous pattern of inheritance. A surprinsing aspect was found regarding patients without a history of cardiovascular disease; these patients were found to carry the mutated gene for factor V Leiden exclusively, thus reaching statistic significance $(\mathrm{Phi}=$

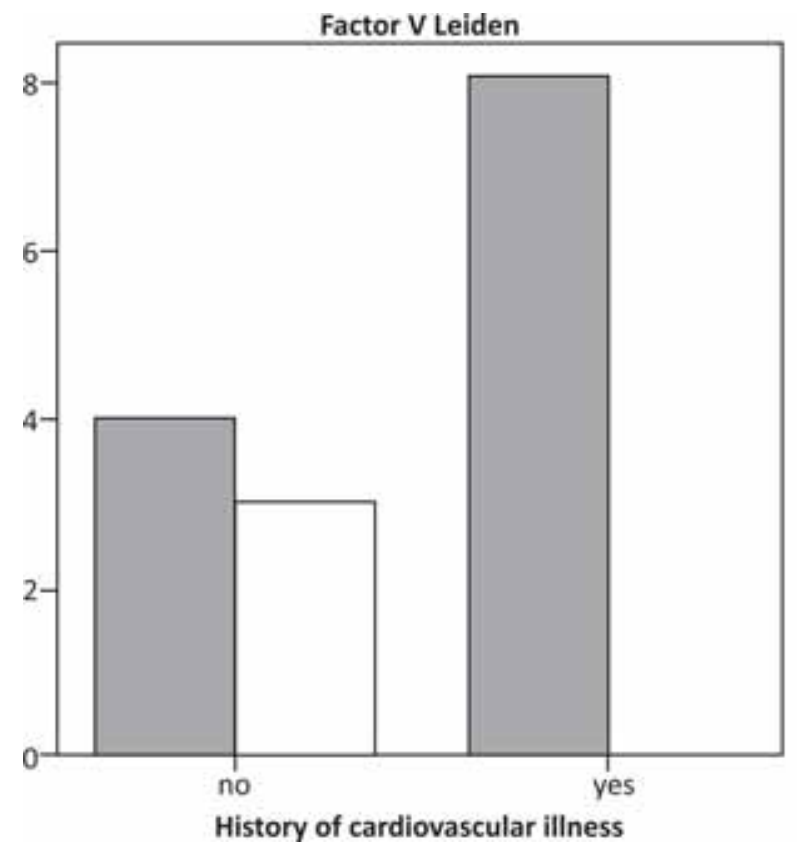

\section{Factor $\mathrm{V}$ Leiden \\ mutation absent \\ $\square$ mutation present}


$0.5, \mathbf{p}=\mathbf{0 . 0 3 8}$ and Chi $(1)=4.3)$. This information is intriguing given the fact that carriers of factor $\mathrm{V}$ Leiden mutation are considered to bear a higher risk for cardiovascular events. The distribution of factor $\mathrm{V}$ Leiden mutation between patients with and without cardiovascular disease can be seen in Graph 3.

Prothrombin mutation was found in $8.7 \%$ of patients included in our study. A surprising fact about our patients' genetic profile was the fact that prothrombin mutation was significantly more frequent among patients without any prior illnesses $(\mathrm{Phi}=$
$0.5, \mathbf{p}=\mathbf{0 . 0 4 7}$, Chi $(1)=4)$. Graph 4 illustrates that only patients without comorbidities exhibited this mutation.

The second most frequent mutation we found among patients with cerebral venous thrombosis was that of the plasminogen activator inhibitor (PAI-1) which was found in over half of our patients (63.6\%). Regarding allele distribution, 42.9\% of carriers were homozygous, as can be seen in Graph 5. PAI-1 was significantly more frequent in men (90\%) than in women (41.7\%), with important effect size $(\mathrm{p}=0.019$, Chi $(1)=5.5$, Phi $=0.5)$.
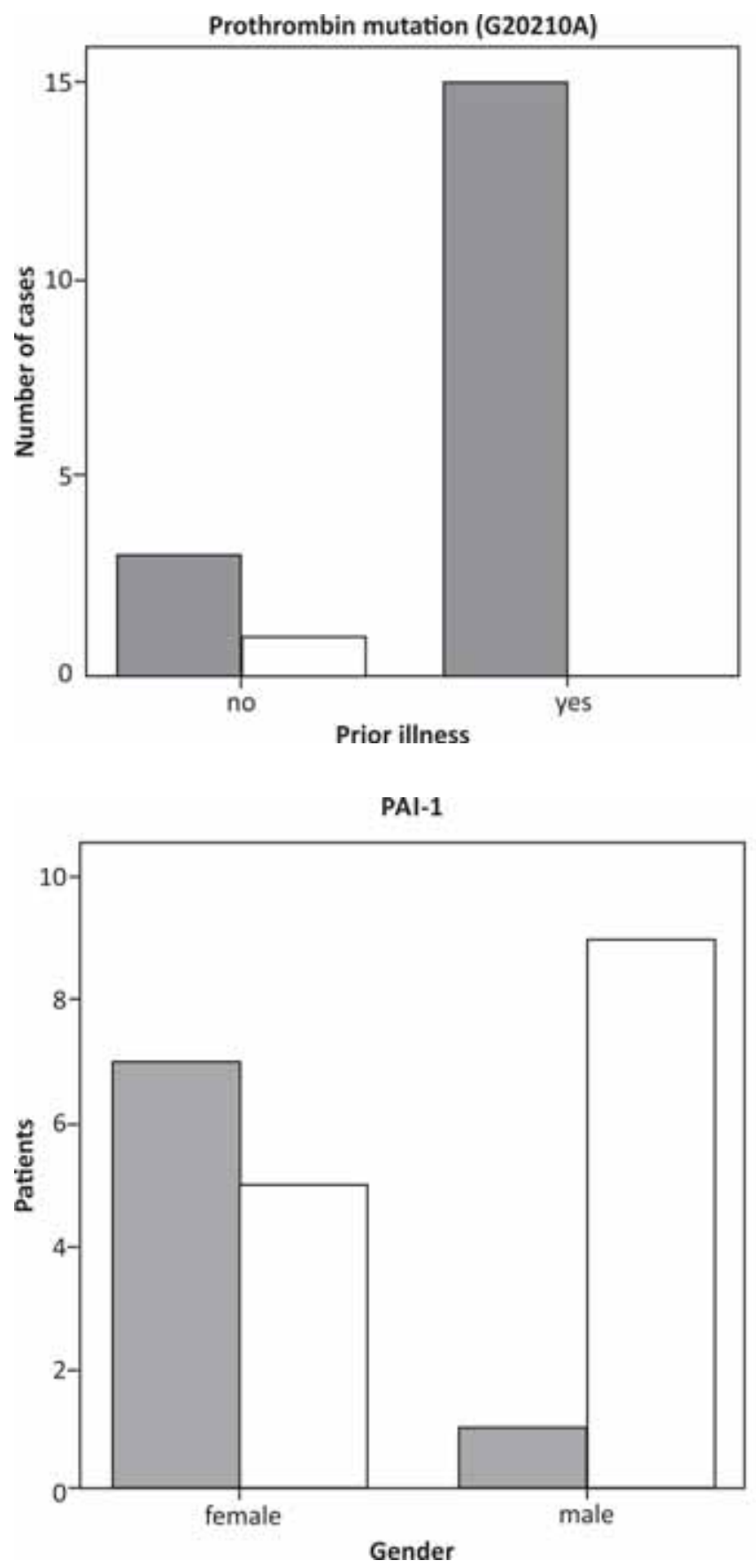

Factor II

$\square$ mutation absent

mutation present

GRAPH 4. Distribution of prothrombin mutation among patients with and without comorbidities

PAI-1

mutation absent

mutation present 


\section{DISCUSSION}

We performed this study in order to show the genetic profile of the Romanian patient with cerebral venous thrombosis. It is the first prospective observational study performed on this disease in Romania. The results of our study concerning hereditary thrombophilia were in agreement with current data in the literature, especially given the fact that all patients presented at least one mutation. The population of patients we enrolled showed clinical and genetic aspects similar to the epidemiologic profile of the patient with cerebral venous thrombosis, regarding age of onset, prior or concomitant infections, presence of prothrombotic factors and favourable outcome. Most patients came from urban areas, but this fact was attributed to easier access to healthcare and high accuracy cerebral imaging studies.

Nevertheless, we found striking differences; for example men and women were equally represented among our patient population, which is atypical considering the fact that women are three times more likely to be affected by cerebral venous thrombosis than men.

Risk factor analysis among our patients showed that the most frequently found predisposing factors were smoking, local infections and the puerperal period. Remarkably, pregnant women and women with hormone replacement therapy were absent from our study, but this could also be due to the fact that our centre does not have an obstetrics ward. Also missing from our study were patients with known malignancies.

Lastly, from a genetic standpoint our study had significant results. All enrolled patients presented at least one mutation, with most patients presenting more than one. The mutations we found for the greatest part were previously considered to pose a low risk of venous thrombosis. The most frequent mutation among our group was MTHFR C677T which by itself does not generate thrombosis, but by raising homocysteine levels is highly thrombogenic. This finding correlates with the overall mean value of homocysteine levels found in our cohort. Another mutation with controversial reports concerning its involvement in the generation of thrombosis is PAI-1, which was highly prevalent among our patients.

\section{CONCLUSIONS}

The presence of hereditary thrombophilia in a young person raises the risk for venous thrombosis. This aspect is unanimous among all recent studies on this subject and our results offer further confirmation. The presence of multiple mutations simultaneously or the association between a hereditary thrombophilia and other established risk factors for venous thrombosis raise the risk of cerebral venous thrombosis exponentially.

The men in our population presented a higher risk for the development of cerebral venous thrombosis than the risk reported in current published data. High overall homocysteine levels could suggest a population predisposition for hypercoagulability due to hyperhomocysteinemia.

The presence of hereditary thrombophilia in all our patients confirms the impact that this pathological state has on the etiology of cerebral venous thrombosis and opens the debate whether we need to devise a primary prevention plan. Furthermore, an ideal timeline should be developed for seeking these mutations and what measures should be taken for each specific mutation so as to have the optimal cost/benefit ratio.

Conflict of interest: none declared Financial support: none declared

\section{REFERENCES}

1. Coutinho J.M., Ferro J.M., Canhao P. et al. Cerebral venous and sinus thrombosis in women. Stroke 2009; 40:2356-61.

2. Tatlisumak T., Jood K., Putaala J. Cerebral Venous Thrombosis. Epidemiology in Change 2016; 47:2169-70.

3. Ferro J.M., Canhao P., Aguiar de Sousa D. Cerebral venous thrombosis. Presse medicale (Paris, France : 1983) 2016; 45:e429-e50.

4. Ferro J.M., Correia M., Pontes C., Baptista M.V., Pita F. Cerebral vein and dural sinus thrombosis in Portugal: 1980-1998. Cerebrovascular diseases (Basel, Switzerland) 2001; 11:177-82.
5. Devasagayam S., Wyatt B., Leyden J., Kleinig T. Cerebral Venous Sinus Thrombosis Incidence Is Higher Than Previously Thought: A Retrospective Population-Based Study. Stroke 2016; 47:2180-2.

6. Ferro J.M., Canhao P., Stam J., Bousser M.G., Barinagarrementeria F. Prognosis of cerebral vein and dural sinus thrombosis: results of the International Study on Cerebral Vein and Dural Sinus Thrombosis (ISCVT). Stroke 2004; 35:664-70.

7. Jellinger K. Handbook of Cerebral Venous Thrombosis. European Journal of Neurology 2009; 16:e38-e. 
8. Popescu Bogdan O. Elemente esențiale de neurologie clinică. Editura Amaltea; 2009:196-9.

9. Young A. Paul Y.H.P. Aportul sanguin al sistemului nervos central: accidentul vascular: Editura Medicală Callisto; 2000.

10. Gomes M.P., Deitcher S.R. Risk of venous thromboembolic disease associated with hormonal contraceptives and hormone replacement therapy: a clinical review. Archives of internal medicine 2004; 164:1965-76.
11. Saposnik G., Barinagarrementeria F., Brown R.D., Jr. et al. Diagnosis and management of cerebral venous thrombosis: a statement for healthcare professionals from the American Heart Association/American Stroke Association. Stroke 2011; 42:1158-92.

12. Einhaupl K., Stam J., Bousser M.G. et al. EFNS guideline on the treatment of cerebral venous and sinus thrombosis in adult patients. Eur J Neurol 2010; 17:1229-35. 